\title{
Multiple Relative Clauses: Ordering and Derivation*
}

\author{
Jeong-Shik Lee \\ (Wonkwang University)
}

\begin{abstract}
Lee, Jeong-Shik. (2017). Multiple Relative Clauses: Ordering and Derivation. Language Research, 53.3, 525-557.
\end{abstract}

\begin{abstract}
There are three types of multiple relative clauses (RCs) in Korean; that is, prenominal, right-dislocated, and split RCs. In this paper, I offer plausible derivations of them and attempt to account for ordering restrictions they display. I propose a couple of distinct ways of forming $\mathrm{RC}$ constructions in Korean, for example, adjunction of $\mathrm{RC}(\mathrm{s})$, complementation of $\mathrm{RC}(\mathrm{s})$, and combination of the two. Concomitantly, the proposal resorts to the head-first hypothesis instead of the usual head-final hypothesis for Korean. In the prenominal and split RCs, a stage level RC precedes an individual level RC in Larson and Takahashi's (2007) term, but in the right-dislocated RCs, free order obtains. An individual level RC may precede a stage level $\mathrm{RC}$ if the former is preceded by a determiner like $k u$ 'the'. I show that different orders of RCs follow from the proposal that to restrict the relative head noun, a deictic quantifier in the relative head noun be activated by a determiner or a stage level $\mathrm{RC}$ in the prenominal position.
\end{abstract}

Keywords: multiple relative clauses, stage level, individual level, deictic quantifier, head-first, head-final

\section{Introduction}

In Korean, in which a relative clause typically appears before the relative head noun, multiple relative clauses can share a relative head noun, as illustrated in the following examples:

* I thank three anonymous reviewers for their helpful comments. Any error, however, is mine. This paper was supported by Wonkwang University in 2017. 
(1) a. [Nay-ka ecey mannan][yenge(-lul) cal hanun] salam-i o-ass-e. I-Nom yesterday met English-Acc well speak person-Nom come-Past-Dec 'The person who speaks English well who I met yesterday came.'

b. [Swunhi-ka ecey mannan][khi(-ka) khun] salam-i nathana-ass-e. S-Nom yesterday met height-Nom tall person-Nom appear-Past-Dec 'The person who is tall who Swunhi met yesterday appeared.'

c. [Nay-ka caknyen-ey chayyonghan][swutasulen] cikwen-i I-Nom last year-in employed talkative employer-Nom get haykotoy-ess-e. fired-Past-Dec

'The talkative employer who I employed last year got fired.'

Examples of the type in (1a) are introduced by Larson and Takahashi (2007); (1b) is adapted from (1a); the AP swutasulen 'talkative'in (1c) is regarded as a reduced relative clause (Kayne 1994).

By contrast, the multiple relative clauses in (1) cannot be freely ordered: if the two relative clauses are switched, the result is considerably degraded or ungrammatical, as observed below.

(2) a. ?*[Yenge(-lul) cal hanun][nay-ka ecey mannan] salam-i o-ass-e.

English-Acc well speak I-Nom yesterday met person-Nom come-Past-Dec 'Lit. Person who speaks English well who I met yesterday came.'

b. ?*[Khi(-ka) khun][Swunhi-ka ecey mannan] salam-i nathana-ass-e. height-Nom tall S-Nom yesterday met person-Nom appear-Past-Dec 'Lit. Person who is tall who Swunhi met yesterday appeared.'

c. *[Swutasulen][nay-ka caknyen-ey chayyonghan] cikwen-i talkative I-Nom last year-in employed employer-Nom get haykotoy-ess-e. fired-Past-Dec

'Lit. Talkative employer who I employed last year got fired.'

Larson and Takahashi (2007) judged examples of the type in (2a) to be bad, which I agree with. It, however, appears that not all speakers of Korean reject this type of examples. In particular, one reviewer reported that Larson and Takahashi's Korean examples of this type are all fine with her/him, although my informants and I can detect the contrast between (1a) and (2a) with little hesitation. It seems thatthe degree of individual level of the predicates involved may be sensed differently from speaker to speaker. I assume the judgments reported here should be considered relative rather than absolute. For this reason, in the ensuing discussion 
I mainly use examples of the type $(1 b, 2 b)$ with the increased degree of individual level of the predicates and reserve those like (1c, 2c) to avoid controversy over the relative clause status of APs as reduced relative clauses.

Following Larson and Takahashi (2007), the contrasts between (1) and (2)may be attributed to the distinction between stage-level relative clauses (henceforth, S-level RCs) and individual level relative clauses (henceforth, I-level RCs). In (1), the preceding relative clauses contain S-level predicates, while the following ones contain I-level predicates. In (2), the opposite situation obtains.1) This then leads to a condition on the surface linear order on multiplerelative clauses in Korean, as stated in (3) (cf. Larson and Takahashi 2007). Below, the sign $>$ indicates that the left element precedes the right one.2)

(3) Multiple relative clauses in Korean appearin the stage level $>$ individual level order.

1) More data, not introduced here, can include multiple relative clauses of the same level, namely, two S-level RCs or two I-level RCs, and they can appear in free order, as observed in Larson and Takahashi (2007). In this paper, I am not concerned with these data displaying no contrasts.

2) One reviewer considered the following Korean examples from Kim (2014: 289, $(21 \mathrm{a}, \mathrm{b}))$ problematic to this condition.

(i) [Mina-ka pyengso aychanghanun][pitulcu-ka pwuless-ten] nolay-nun leylipi-ita.

M-Nom usually like-to-sing Beatles-Nom sang song-Top Let-it-be-be

'The song that Mina usually likes to sing that Beatls sang is Let-it-be.'

(ii) ??[Pitulcu-ka pwuless-ten][Mina-ka pyengso aychanghanun] nolay-nun leylipi-ita. Beatles-Nom sang M-Nom usually like-to-sing song-Top Let-it-be-be

'The song that Mina usually likes to sing that Beatls sang is Let-it-be.'

This reviewer's point runs as follows: in (i) the I-level RC precedes the S-level RC, but (i) is good; in (ii) the S-level RC precedes the I-level RC, but (ii) is degraded (while Kim's 2014 judgment is ??, as indicated, this reviewer's judgment is ?). But it is not clear whether the RC [pitulcu-ka pwuless-ten] above is strictly stage-level. Rather, it may be I-level in that the song can refer to the popular one that is known to have been sung by a famous group of singers. Also, the degree of individual level of the RC [Mina-ka pyengso aychanghanun] seems somewhat low. Although the adverb pyengso 'usually' contributes to individual level property, the RC here can also be understood to involve frequent activity of singing the song, hence more like an S-level predicate. Thus, the above examples may not clearly represent a problem with the current analysis. Kim (2014: 288, (20a,b)) also reports the same contrast as that between (1a) and (2a), with slightly different examples, where an S-level RC must precede an I-level RC. 
According to Larson and Takahashi (2007), this condition holds across languages such as Chinese, Japanese and Turkish as well as Korean.

Surprisingly, if a determiner like $k u$ 'the' comes in front, the badness of the examples in (2) disappears, as seen below.3)

(4) a. [Ku [yenge(-lul) cal hanun][nay-ka ecey mannan] salam]-i o-ass-e.

the English-Acc well speak I-Nom yesterday met person-Nom come-Past-Dec

'The person who speaks English well who I met yesterday came.'

b. [Ku [khi(-ka) khun][Swunhi-ka ecey mannan] salam]-i

the height-Nom tall S-Nom yesterday met person-Nom

nathana-ass-e.

appear-Past-Dec

'The person who is tall who Swunhi met yesterday appeared.'

c. [Ku [swutasulen][nay-ka caknyen-ey chayyonghan] cikwen]-i

the talkative I-Nom last year-in employed employer-Nom get

haykotoy-ess-e.

fired-Past-Dec

'The talkative employer who I employed last year got fired.'

The above observation makes Larson and Takahashi's condition in (3) ineffective. I set aside a question as to why this happens until section 3. At this point, I merely suspect that in (2), the initial I-level (reduced) RCs do not sufficiently restrict the relative head noun, in contrast with the initial S-level RCs as in (1), and that in (4), the determiner $k u$ 'the' helps do so. 4 )

I also observe that one of the multiple relative clauses can be right-dislocated with a limit, which I call split RCs, as seen in (5). In the following

3) Kim (2016) also observes this fact. She (p. 176) considers examples like (i) similar to (4c), where $k u$ is treated as an atypical cognitive demonstrative, denoted as $K U$, that precedes an $\mathrm{AP}$ rather than $\mathrm{RC}$, and she (p. 182) claims this $K U$ forms a syntactic unit with the following $\mathrm{AP}$.

(i) $\left[\mathrm{ku}\right.$ tokcaycek], [e mincwucwuuy-lul thanaphan], kwuncwu $\mathrm{e}_{\mathrm{i}}$ that tyrannical democracy-Acc oppressed monarch 'that tyrannical monarch who oppressed democracy' (Kim 2016: 176, (2b))

I assume that the $\mathrm{ku}$ in $(4 \mathrm{a}, \mathrm{b})$ is different from Kim's KU in that it precedes an RC rather than an AP. I do not assume that the ku forms a constituent with the following $\mathrm{AP}$ or RC, either.

4) A different observation is made from Chinese; that is, despite the presence of a determiner, the I-level > S-level order is not allowed in Chinese (see Del Gobbo 2005, Lin 2008). 
discussion, right-dislocated constructions are called RDCs, for short.5)

(5) a. [Swunhi-ka ecey mannan] salam-i nathana-ass-e [khi(-ka) khun].

S-Nom yesterday met person-Nom appeared height-Nom tall

'The person who Swunhi met yesterday appeared who is tall.'

b. ?*[Khi(-ka) khun] salam-i nathana-ass-e [Swunhi-ka ecey mannan].

height-Nom tall person-Nom appeared S-Nom yesterday met.

'Lit. Person who is tall appeared who Swunhi met yesterday.'

It is seen that while an I-level RC can be right-dislocated, an S-level $\mathrm{RC}$ cannot. (5b) is just as bad as (2b). For some speakers, however, (5b) seems not as bad as (2b). Despite this, I basically assume that there exists a significant contrast between (5a) and (5b). Again, if a determiner like $k u$ 'the'comes in front, the badness of the example in (5b) disappears, as seen below.

(6) $[\mathrm{Ku}[\mathrm{khi}(-\mathrm{ka}) \quad \mathrm{khun}] \mathrm{salam}]-\mathrm{i}$ nathana-ass-e [Swunhi-ka ecey mannan]. the height-Nom tall person-Nom appeared S-Nom yesterday met. 'The person who is tall appeared who Swunhi met yesterday.'

Interestingly, when relative clauses are multiply right-dislocated, the contrast between (1b) and ( $2 b)$ and that between (5a) and (5b)disappear.

(7) a. Ku salam-i nathana-ass-e [khi(-ka) khun] [Swunhi-ka ecey mannan]. the person-Nom appeared height-Nom tall S-Nom yesterday met 'The person appeared who is tall who Swunhi met yesterday.'

b. Ku salam-i nathana-ass-e [Swunhi-ka ecey mannan] [khi(-ka) khun]. the person-Nom appeared S-Nom yesterday met height-Nom tall 'The person appeared who Swunhi met yesterday who is tall.'

The contrast in (5) may follow from the condition in (3) allowing the stage level > individual level order only. But the loss of the contrast in (7) casts doubt on the validity of this condition and requires a separate explanation. Here, the RCs considered so far are intended to be restrictive; in particular, the right-dislocated RCs in $(7 a, b)$ are also intended to be

5) Since right-dislocation matches better with a spoken style, the declarative verbal ending marker -e is used instead of the typical written style -tahere and throughout the paper thereof. 
restrictive.6) On the other hand, if the determiner $k u$ 'the' is absent in $(7 a, b)$, the examples become bad.

(8) a. *Salam-i nathana-ass-e [khi(-ka) khun] [Swunhi-ka ecey mannan]. person-Nom appeared height-Nom tall S-Nom yesterday met

'Lit. Person appeared who is tall who Swunhi met yesterday.'

b. *Salam-i nathana-ass-e [Swunhi-ka ecey mannan] [khi(-ka) khun]. person-Nom appeared S-Nom yesterday met height-Nom tall 'Lit. Person appeared who Swunhi met yesterday who is tall.'

Thus, it appears that something other than the condition in (3) is at work.

In this paper, I attempt to resolve the (conflicting) contrasts observed so far under a new analysis. Although RDCs in Korean have received a double clause approach under the traditional head-final structure in most studies (see Chung 2009, Park and Kim 2009, among others), I will argue against this analysis and other minor approaches in section 2. As an alternative, I propose a single clause approach adopting the head-first hypothesis for Korean in section 3. Derivations of different order patterns of multiple relative clauses are illustrated under the head-first hypothesis in Korean. Adapting Larson and Takahashi (2007), ordering restrictions between multiple relative clauses are captured in a way different from them. I also offer a proposal for ways of restricting relative

6) That a right-dislocated RC can be restrictive is shown by the following binding fact.

(i) [Motun haksayng $]_{\mathrm{i}-\mathrm{i}}$ ta han yeca-wa chwum-ul chwu-ess-e [ku $\mathrm{k}_{\mathrm{i}}-\mathrm{lul}$ cohaha-nun]. Every student-Nom all a girl-with dance-Acc danced he-Acc like-Rel 'Every student danced with a girl that likes him.

Here every binds into the pronoun $k u$ in the relative clause, which is known to be a property ofthe restrictive relative clauses (Safir 1986). Also, the RDed RC still remains at LF with VP-ellipsis (or NP-ellipsis), which is another property of the restrictive relative clauses (Del Gobbo 2005: 301).

(ii) a. Chelswu $\mathrm{i}_{\mathrm{i}} \mathrm{ka}$ han yeca-lul manna-ass-e [caki $\mathrm{i}_{\mathrm{i}}-\mathrm{lul}$ cohahanun]

Chelswu-Nom one woman-Acc met self-Acc love

'Chelswu met a woman who loves him.'

b. Yengswu-to [ e ] manna-ass-e.

Yengswu-also met

'Yengswu also met a woman who loves him.'

$([\mathrm{e}]=$ [VP han yeca-lul manna-[caki-lul cohahanun] $])$

In addition, the subject can bind the anaphor in the RDed RC in (iia), which is usually found with the restrictive relatives. See Del Gobbo (2005) for more related discussion. 
clauses in Korean. Finally, section 4 offers summary and conclusion.

\section{A Review of Previous Approaches}

This section examines previous approaches to see if they can properly derive types of multiple relative constructions introduced in the previous section. I will mainly consider the most popular double clause approach to RDCs coupled with Move and Delete, and then the minor mono-clausal approach coupled with rightward or leftward movement. I eventually argue that they are not successful, revealing that the head-final hypothesis those analyses adopt should be blamed.

\subsection{Double Clause Approach}

According to Chung (2009), a simple RDC like (9) would bederived from the double clause source followed by some complex process of ellipsis, as illustrated in (10). The discussion below assumes the usual head-final hypothesis in Korean with the structure (10d); more generally, the base order in Korean is Subject $>$ Complement $>$ Head, or SOV.

(9) $\mathrm{Ku}$ salam-i nathana-ass-e [Swunhi-ka ecey mannan]. the person-Nom appeared S-Nom yesterday met 'The person appeared who Swunhi met yesterday.'

(10) a. [[Swunhi-ka ecey mannan] ku salam-i nathana-ass-e]

S-Nom yesterday met the person-Nom appeared [[Swunhi-ka ecey mannan] ku salam-i nathana-ass-e]

b. [[Swunhi-ka ecey mannan] ku salam-i nathana-ass-e] [[Swunhi-ka ecey mannan] ku salam-i nathana-ass-e]

c. [[Swunhi-ka ecey mannan] ku salam-i nathana-ass-e] [[Swunhi-ka ecey mannan] ku salam-i nathana-ass-e] 'Intended. The person who Swunhi met yesterday appeared.' d. [DP RC [D [NP [ N ]]]]

(10a) represents a double clause source; in (10b), backward ellipsis of 
the relative clause is applied in the preceding clausal conjunct; in (10c), ellipsis of the following clausal conjunct is applied except the relative clause.7)8) In (10a) the determiner $k u$ 'the' appears after the prenominal relative clause, which also constitutes a restrictive relative clause in the sense of Del Gobbo (2005).9)

It is seen that in (10a), the same clause is doubled to produce a coordinate structure. This kind of clause doubling may be needed for the purpose of emphasis or confirmation. However, the backward ellipsis, applied in (10b), is not actually attested in regular coordinate clausal structures in Korean (see Lee2009, 2011a).
(11) a. [[Swunhi-ka ecey
mannan] $\mathrm{ku}$ salam-i
khuta] kuliko
S-Nom yesterday met the person-Nom tall and
[[Swunhi-ka ecey mannan] ku salam-i nalssinhata]
S-Nom yesterday met the person-Nom slim
b. *[Swunhi-ka eeey mannan] ku salam-i khuta] kuliko
[[Swunhi-ka ecey mannan] ku salam-i nalssinhata]
'Intended. The person who Swunhi met is tall, and the person is slim.'
c. [[Swunhi-ka ecey mannan] ku salam-i khuta] kuliko
[Ewunhi-ka ecey mannan] ku salam-i nalssinhata]
'The person who Swunhi met is tall, and the person is slim.'

The contrast between (11b) and (11c) tells us that only forward ellipsis is allowed. This means that the backward ellipsis in (10b) is quite exceptional, being allowed in the case of clause doubling only. Note that the

7) In passing, I point out that the elliptic relative clause, being a clausal modifier, cannot be regarded as pro, which is usually posited for the gap in the preceding clause in RDCs in Chung (2009).

8) In (10c) (and (12c, 13c) below as well) the elliptic portion of the second conjunct clause in fact is not a constituent, and thus, the relative clause is assumed to have raised to the clause initial position so that the remaining part can be deleted as a whole constituent. Island violation incurred thereof may be hidden by Repair by Ellipsis.

9) One might say that this RC is not a restrictive RC but an appositive (Kim 1996) or supplementary (Kim 2014). However, this may not be correct in that this RC is certainly restrictive (see also Del Gobbo 2005 for related discussion in detail). All the RCs considered in this paper, prenominal or right-dislocated, are intended to be restrictive. In addition, the restrictive $\mathrm{RC}$ before the determiner in the configuration of (10d) can also be individual level (see also Lin 2008 for this fact in Chinese), unlike in Larson and Takahashi (2007), according to which an I-RC is an NP-modifier (see (29) in section 3.2.1.). 
doubled clause has to be deleted again except the RDed element, as seen in (10c). This is inconsistent with the spirit of Minimal Computation (Chomsky 2008). A shorter derivation that involves no such redundancy would clearly be more economical. As mentioned above, consider also that clause doubling is usually applied for the purpose of emphasis or confirmation ofthe proposition of the clause. The RDed element in (9), however, contributes neither emphasis nor confirmation originally intended. So the clause doubling in (10a) is applied only for the purpose of getting the RDed element, which renders it ad hoc. This indicates that (9) may well be derived from a different source than the double clause source. If so, the exceptionality of the backward ellipsis in (10b) would also be eliminated.

In short, it is unclear whether the double clause approach can apply to RDCs like (9). This is mainly because the backward ellipsis in this analysis is not sufficiently motivated. This point will be further strengthened when multiple relative clause constructions are considered below.

Let us consider the contrast from (5), repeated below.

(5) a. [Swunhi-ka ecey mannan] salam-i nathana-ass-e [khi(-ka) khun]. S-Nom yesterday met person-Nom appeared height-Nom tall 'The person who Swunhi met yesterday appeared who is tall.'

b. ?*[Khi(-ka) khun] salam-i nathana-ass-e[Swunhi-ka ecey mannan]. height-Nom tall person-Nom appeared Swunhi yesterday met 'Lit. Person who is tall appeared who Swunhi met yesterday.'

Under the double clause approach, (5a) will be derived (assume the structure in (12d)), as illustrated below.

(12) a. [[Swunhi-ka ecey mannan][khi(-ka) khun] salam-i nathana-ass-e] [[Swunhi-ka ecey mannan][khi(-ka) khun] salam-i nathana-ass-e]

b. [[Swunhi-ka ecey mannan] [khi(-ka) khun] salam-i nathana-ass-e] [[khi(-ka) khun][Swunhi-ka ecey mannan] [khi(-ka) khun] salam-i nathana-ass-e]

c. [[Swunhi-ka ecey mannan] [khi(-ka) khun] salam-i nathana-ass-e] [[khi(-ka) khun] [Swunhi-ka eeey mannan] [khi(-ka) khun] salam-i nathana-ass-e]]

d. [DP S-RC [I-RC [D [NP [ N ] ] ]]] $]^{10)}$

10) The number of multiple RCs may be more than two, and each relative clause may appear in the Spec of a certain functional head, forming a cartographic structure (cf. 
In (12b), the unwanted backward ellipsis has applied in the first clausal conjunct, which is inevitable under the double clause approach, and the I-level RC has fronted in the second clausal conjunct to remain as an $\mathrm{RD}$ material; in (12c), the second clausal conjunct has undergone ellipsis except the fronted I-level RC. As a result, the surface (5a) is derived. Notice, here, that the second clausal conjunct in (12b) is in fact bad, which is confirmed in (2b) in which an I-level RC cannot precede an S-level RC. This problem may be fixed by further ellipsis in (12c), which eliminates the source of the problem, the so called Repair by Ellipsis.

Now let us derive $(5 b)$ under the double clause approach (assume the same structure (12d)). Its derivation is pretty much the same as that of (5a), as illustrated below.

(13) a. [[Swunhi-ka ecey mannan][khi(-ka) khun] salam-i nathana-ass-e] [[Swunhi-ka ecey mannan][khi(-ka) khun] salam-i nathana-ass-e]

b. [[Swunhi-ka ecey mannan] [khi(-ka) khun] salam-i nathana-ass-e] [[Swunhi-ka ecey mannan] [khi(-ka) khun] salam-i nathana-ass-e]

c. [ESunhi-ka eey mand [khi(-ka) khun] salam-i nathana-ass-e] [[Swunhi-ka ecey mannan] [ $[\mathrm{khi}(-\mathrm{ka})$ khun] salam-i nathana-ass-e]]

In (13b), the backward ellipsis has applied in the first clausal conjunct, just as in (12b); in (13c) the S-level RC has fronted to remain as an $\mathrm{RD}$ material in the second clausal conjunct, which has further undergone ellipsis except the preceding relative clause, just as in (12c). As a result of this deletion, the wrong surface (5b) is incorrectly derived. Unfortunately, the double clause approach cannot block this derivation.11)

As for the contrast in (5), one might resort to thecondition on the surface linear order on multiple relative clauses, as stated in (3), repeated below.

Kim 2014 for one such structure). I leave this elaboration for another study.

11) Kim and Park's (2010) scattered deletion, which they call Conjunction Reduction (CR), faces the same problem. Their CR approach assumes non-constituent in situ deletion without movement, and thus, in (13c), for example, deletion applies to the strike-throughed non-constituent string in the second conjunct clause (in addition to backward deletion of the RC in the first conjunct clause). 
(3) Multiple relative clauses in Korean appear in the stage level > individual level order.

Although this condition appears to deal with the contrast in (5), it undesirably excludes the example in (6), repeated below, because of the ordering that it prohibits.

(6) $[\mathrm{Ku}[\mathrm{khi}(-\mathrm{ka}) \quad$ khun] salam]-i nathana-ass-e [Swunhi-ka ecey mannan].

the height-Nom tall person-Nom appeared S-Nom yesterday met.

'The person who is tall appeared who Swunhi met yesterday.'

It does not work, either, when RDed multiple relative clauses are taken into consideration--they may appear in either order, as seen in (7), repeated below.

(7) a. Ku salam-i nathana-ass-e [khi(-ka) khun] [Swunhi-ka ecey mannan]. the person-Nom appeared height-Nom tall S-Nom yesterday met 'The person appeared who is tall who Swunhi met yesterday.'

b. Ku salam-i nathana-ass-e [Swunhi-ka ecey mannan] [khi(-ka) khun]. the person-Nom appeared S-Nom yesterday met height-Nom tall 'The person appeared who Swunhi met yesterday who is tall.'

Larson and Takahashi (2007) observed that postnominal multiple relative clauses in English may appear in either order.12)

(14) a. the person [who smokes][who I met] (individual $>$ stage) b. the person [who I met][who smokes] (stage > individual)

Thus, similarly to English, it may be said that right-dislocated RCs in (7) is not subject to the ordering condition. As observed in (5b), however, a right-dislocated RC is certainly involved in this matter. It is also noted that under the double clause analysis, the multiple relative clauses in (7) are not really postnominal but prenominal, as seen in $(15 a, b)$. As the ordering condition in (3) forces only one order, namely, the prenominal stage $>$ individual order, the order in the second conjunct clause in (15a)

12) But they did not report RDed multiple relative clauses like $(7 a, b)$ in Korean. 
(i.e., individual $>$ stage) should not be allowed, contrary to fact (cf. (2b)), while the one in (15b) (i.e., stage $>$ individual) is (cf. (1b)).

(15) a. [Ku salam-i nathana-ass-e][[khi(-ka) khun] [Swunhi-ka ecey mannan] ku salam-i nathana-ass-e]

b. [Ku salam-i nathana-ass-e] [[Swunhi-ka ecey mannan] [khi(-ka) khun] ku salam-i nathana-ass-e]

Note also that (15a) cannot be salvaged by a mechanism like Repair by Ellipsis under the double clause analysis. This is because the source of the problem lies outside the elliptic site.

In sum, the double clause approach cannot properly handle the distributions of multiple relative clausesin Korean. In what follows, alternative analyses will be explored.

\subsection{Mono-clausal Rightward Movement Approach}

Upon the failure of the double clause approach, a single clause approach is worth pursuing. For the ensuing discussion, the examples from $(5 a, b)$, repeated as $(16 a, b)$, and those from $(1 b, 2 b)$, repeated as $(17 a, b)$, are reconsidered. The discussion below also assumes the head-final hypothesis with the structure (17c); more generally, the base order is Subject > Complement $>$ Head, or SOV, in Korean.

(16) a. [Swunhi-ka ecey mannan] salam-i nathana-ass-e [khi(-ka) khun]. S-Nom yesterday met person-Nom appeared height-Nom tall 'The person who Swunhi met yesterday appeared who is tall.'

b. ?*[Khi(-ka) khun] salam-i nathana-ass-e [Swunhi-ka ecey mannan]. height-Nom tall person-Nom appeared S-Nom yesterday met

'Lit. Person who is tall appeared who Swunhi met yesterday.'

(17) a. [Swunhi-ka ecey mannan][khi(-ka) khun] salam-i nathana-ass-e. S-Nom yesterday met height-Nom tall person-Nom appear-Past-Dec 'The person who is tall who Swunhi met yesterday appeared.'

b. ?*[Khi(-ka) khun][Swunhi-ka ecey mannan] salam-i nathana-ass-e. height-Nom tall S-Nom yesterday met person-Nom appear-Past-Dec

'Lit. Person who is tall who Swunhi met yesterday appeared.'

c. [DP S-RC [I-RC [D [NP [ N ] ]]]] 
If the multiple relative clause construction in (16a) is a single sentence, it can be derived from the base (17a) via rightward movement of the I-level RC to the sentence final position, as represented in (18a). Also, (16b) can be derived from (17a) via rightward movement of the S-level $\mathrm{RC}$ to the sentence final position, as shown in (18b).

(18) a. [[Swunhi-ka ecey mannan] $t_{i}$ salam]-i nathana-ass-e [khi(-ka) khun]i.

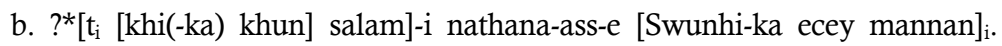

In contrast with (18a), however, (18b) is bad.

Where does the contrast come from? It cannot be simply ascribed to structural reasons. If the linear sequence in unmarked order reflects hierarchy, the contrast between (17a) and (17b) indicates that the S-level RC is higher than the I-level RC here. However, in contrast with (17a), the marked order in (16b) is bad although the S-level RC, right-adjoined here (see (18b)), is higher than the I-level RC; in contrast with (17b), the marked order in (16a) is good although the I-level RC, right-adjoined here (see (18a)), is higher than the S-level RC. Thus, a structural account is not successful. As observed in (7), repeated in the previous section, multiple relative clauses can be right-dislocated in either order. In particular, ifthe badness of $(16 b, 17 b)$ is due to the precedence of the I-level RC to the S-level RC, it remains puzzling why (7a) is good although the I-level $\mathrm{RC}$ precedes the S-level RC here. This implies that the distribution of multiple relative clauses displaying conflicting contrasts in (7) and (16, 17) cannot be simply accounted for by the surface linear order condition offered in (3). In fact, right-dislocating one RC out of the complex DP in (18a,b) (see (17c)) should yield an island violation. Thus, (18a) must be excluded, too, contrary to fact. In short, the rightward movement analysis under the single clause approach is not tenable.13)

13) Before proceeding, another single clause approach realized by repeated leftward movement under the head-last hypothesisis briefly considered. When derivation of an example like (7a), repeated as (i) below, is considered, as given in (ii) below, this analysis turns out to be less likely to work.

(i) Ku salam-i nathana-ass-e [khi(-ka) khun] [Swunhi-ka ecey mannan] (= (7a)) the person-Nom appeared height-Nom tall Swunhi-Nom yesterday met

'The person who is tall who Swunhimet yesterday appeared.' 
Unfortunately, it is seen that operations like clause doubling followed by Move and Delete and rightward or leftward movement suggested for the derivation of the RDCs in question are rendered futile. It is obvious that this is owing to the head-final hypothesis.

\section{Proposal}

\subsection{The Head-first Hypothesis}

Now that the head-final approach fails, I will try a head-first approach. Under the head-first hypothesis, a relative clause moves from the postnominal base position to the prenominal position to derive the typical prenominal relative construction in Korean, as roughly illustrated in (19) (see Kayne 1994 for relevant discussion). ${ }^{14)}$

(19) a. salam [Swunhi-ka ecey $e_{i}$ mannan]

b. [Swunhi-ka ecey $e_{i}$ mannan $]_{j}$ salam $_{i} t_{j}$

I will offer derivations of the multiple relative clauses under the above

(ii) a. [Swunhi-ka ecey mannan] $[\mathrm{khi}(-\mathrm{ka}) \mathrm{khun}] \mathrm{ku}$ salam-i nathana-ass-e

b. [khi(-ka) khun $]_{j}[\text { Swunhi-ka ecey mannan }]_{\mathrm{i}}\left[\mathrm{t}_{\mathrm{i}} \mathrm{t}_{\mathrm{j}}\right.$ [ku salam]]-i nathana-ass-e]

c. $\left[\left[\mathrm{t}_{\mathrm{i}} \mathrm{t}_{\mathrm{j}}[\mathrm{ku} \text { salam]]-i nathana-ass-e }]_{\mathrm{k}}\left[[\mathrm{khi}(-\mathrm{ka}) \text { khun }]_{j}[\text { Swunhi-ka ecey mannan }]_{\mathrm{i}} \mathrm{t}_{\mathrm{k}}\right]\right.\right.$ $(=>(7 a))$

The surface (i) will be derived from the base (iia); two relative clauses individually move to the left as seen in (iib), involving island violations; the remaining clause again moves to the left as seen in (iic). Note, however, that the surface of (iib) is as bad as (2b). Thus, it is not clear how the leftward movement of the remaining clause as illustrated in (iic) cancels the badness of (iib) resulting from island violations as well as wrong ordering.

14) Somali is an SOV language, like Korean, and displays surface postnominal RCs as in (19a). Chinese serves as a real case in point for the derivation in (19b), the language being widely regarded as a head-first language, but its relative clause is prenominal, as seen in (i).

(i) [wo zuotian kanjian de] ren

I yesterday meet DE man

'the man that I met yesterday.'

Why English RCs cannot be prenominal is another matter, which can be explored in terms of strict head-firstnature of this language. 
head-first hypothesis in detail in the next section. More generally, I assume that the base order is Subject > Head > Complement, or SVO, for all other phrases in Korean, adopting the skeletal head structure, as given in (20), which is not fully motivated and illustrated here for space reasons (see Lee 2011b for inner AspP/MP, see also Lee 2009, 2010, 2011a for relevant discussions).

$$
\text { (20) } \mathrm{T}-\mathrm{v}-\mathrm{M}(\mathrm{ood}) \text { - Asp(-Hon) - Vroot }
$$

Under the current approach, I assume that multiple relative clauses may appear in either order in the postnominal base position, as seen in $(21 a, b)$ (see also $(14 a, b)$ from English). If the relative head is separated from the RCs, the right-dislocated RCs will be derived, as realized in $(7 \mathrm{a}, \mathrm{b})$.

(21) a. salam ${ }_{i}\left[e_{i}\right.$ khi(-ka) khun] [Swunhi-ka ecey ei mannan] b. salam ${ }_{i}$ [Swunhi-ka ecey $e_{i}$ mannan] [ $e_{i}$ khi(-ka) khun]

If one of the RCs moves to the prenominal position and the other is stranded or right-dislocated, split RC construction will be derived, as seen in (22a); if both of the RCs move to the prenominal position, prenominal $\mathrm{RC}$ construction will be derived, as seen in (22b).

(22) a. [Swunhi-ka ecey $e_{i}$ mannan] salam $\mathrm{m}_{\mathrm{i}} \mathrm{i}$ nathanaa-ass-e [e $\left.\mathrm{e}_{\mathrm{i}} \mathrm{khi}(-\mathrm{ka}) \mathrm{khun}\right]$ b. [Swunhi-ka ecey $e_{i}$ mannan] [e $e_{i}$ khi(-ka) khun] salam $m_{i}-i$ nathana-ass-e

As noted before, however, only an S-level RC can precede an I-level $\mathrm{RC}$ when either or both of them move to the prenominal position, as seen in the contrast between (22) and (23).

(23) a. ?* $\left.\mathrm{e}_{\mathrm{i}} \mathrm{khi}(-\mathrm{ka}) \mathrm{khun}\right]$ salam $\mathrm{i}_{\mathrm{i}} \mathrm{i}$ nathana-ass-e [Swunhi-ka ecey $\mathrm{e}_{\mathrm{i}}$ mannan] b. ?*[e $e_{i}$ khi(-ka) khun] [Swunhi-ka ecey $e_{i}$ mannan] salam $m_{i}-i$ nathana-ass-e

So a question arises as to how free ordering between an S-level RC and an I-level RC can be allowed in the right-dislocated position on one hand, and why only an S-level RC appears or precedes an I-level RC in the 
prenominal position on the other hand. If this question can be answered in any reasonable way, the leftward movement approach under the head-first hypothesis will be tenable.

In what follows, I will show that it is so and attempt to account for the intriguing contrasts at issue. As a preparatory step, consider the examples in $(24,25)$, in whichthe outermost brackets represent the intended constituency of the DP that can function as subject or object in typical predicate-final contexts. ${ }^{15) 16)}$

(24) a. *[ku salam [Swunhi-ka ecey mannan] [khi(-ka) khun]]-i/ul b. *[ku salam [khi(-ka) khun] [Swunhi-ka ecey mannan]]-i/ul c. *[ku salam-i/ul [Swunhi-ka ecey mannan] [khi(-ka) khun]] d. *[ku salam-i/ul [khi(-ka) khun] [Swunhi-ka ecey mannan]]

(25) a. *[[Swunhi-ka ecey mannan] salam [khi(-ka) khun]]-i/ul b. *[[khi(-ka) khun] salam [Swunhi-ka ecey mannan]]-i/ul c. *[[Swunhi-ka ecey mannan] salam-i/ul [khi(-ka) khun]] d. *[[khi(-ka) khun] salam-i/ul [Swunhi-ka ecey mannan]]

It is observed that the whole DP or the relative head noun phrase cannot take the nominative case marker $-i$ or the accusative case marker-ul. The modifying RC, being adjectival, cannot directly take a case marker (see

15) In (24) the determiner ku 'the' appears before the head noun salam. In (25) this determiner is absent, but it may appear before or after the prenominal RC, both cases constituting restrictive relative clauses, as mentioned before.

16) I leave discussion of appositive relative clauses in Korean for another study. One such example is provided below for the purpose of distinction from the restrictive relative clause (App.Comp $=$ appositive relative complementizer).

(i) a. Ku salam-un, Swunhi-ka ecey manna-ass-nuntey, tasi nathana-ass-e. the person-Top, S-Nom yesterday met-App.Comp, again appeared 'The person, who Swunhi met yesterday, appeared again.'

b. ?Swunhi-ka ecey manna-ass-nuntey, ku salam-i tasi nathana-ass-e.

c. ?Ku salam-i tasi nathana-ass-e, Swunhi-ka ecey manna-ass-nuntey.

As shown above, the nuntey-appositive can appear in the postnominal position most naturally, in which case it behaves as a parenthetical in a broader sense, compared with the fronted one in (ib) and the right-dislocated one in (ic), with the nominative case marker - $i$ instead of the topic marker -un on the subject in both cases. In addition, appositive clauses in Korean can also appear as different adverbial clauses. 
(24a,b), (25a,b)), and the relative head noun phrase cannot be assigned case inside the DP (see $(24 c, d),(25 c, d))$. Owing to the presenceof the postnominal $\mathrm{RC}(\mathrm{s})$, it appears that the relative head noun cannot directly take its proper case affix. This can be stated as a morphological problem. In other words, an RC or multiple RCs must not appear right after the relative head noun in Korean. This implies that the postnominal RC(s) must vacate its/their original position(s), so that the relative head noun can safely take its case affix. This vacation can be achieved by raising the base postnominal $\mathrm{RC}(\mathrm{s})$ to the prenominal position, as manifested in $(17 a, b)$, in which case ordering restriction is observed, by right-dislocating the base postnominal $\mathrm{RC}(\mathrm{s})$, as manifested in $(7 \mathrm{a}, \mathrm{b})$, in which case ordering restriction is not observed somehow, or by raising one relative clause to the prenominal position and the other to the right-dislocated position, as seen in $(16 a, b)$, in which case ordering restriction is observe d. ${ }^{17)}$ So the presence or the absence of ordering restrictions in those cases remains to be explained.

\subsection{Three Types of RCs: Ordering and Derivation}

In this section, I propose a few different ways of forming RCs that can be used to produce three types of RCs in Korean: prenominal, right-dislocated, and split RCs. They will be realized in detail in the course of elaborating modes of merging relative clauses with a relative head noun.

\subsubsection{Right-dislocated RCs}

To restrict the relative head noun, RCs need to be merged with this head noun. In the case of right-dislocated RCs in $(7 \mathrm{a}, \mathrm{b})$, repeated below, the relative head DP ku salam 'the person' should be separable from the merged RCs.

17) The sequences in $(24 c, d)$ and $(25 c, d)$ may appear in the right-dislocated position, in which case the relative head individually raises to a higher position for Case or other reasons and later reordering renders the head adjacent to the RCs (see section 3.2.1. for relevant derivations). 
(7) a. Ku salam-i nathana-ass-e [khi(-ka) khun] [Swunhi-ka ecey mannan]. the person-Nom appeared height-Nom tall S-Nom yesterday met 'The person appeared who is tall who Swunhi met yesterday.'

b. Ku salam-i nathana-ass-e [Swunhi-ka ecey mannan] [khi(-ka) khun]. the person-Nom appeared S-Nom yesterday met height-Nom tall 'The person appeared who Swunhi met yesterday who is tall.'

One possibility to realize this fact under the current head-first approach is that RCs, labeled as \&P in (26), are right-adjoined to the head DP ku salam, as represented below (cf. Bach and Cooper 1978, Aoun and Li 2003, Del Gobbo 2005 for this adjunction structure for other languages).

(26) a. [DP [DP ku [NP [N salam]]] [\&P [CP Op [Swunhi-ka ecey e mannan]] \& [CP Op [khi(-ka) khun]]]]

b. [DP [DP ku [NP [N salam]]] [\&P [CP Op [khi(-ka) khun]] \& [CP Op [Swunhi-ka ecey e mannan]]]]

Above, each RC hostsa relative operator Op. The relative head DP is then separated from the RCs to yield the right-dislocated RCs in $(7 a, b)$, repeated above.

When there occur multiple relative clauses in the postnominal position, I assume that the first relative clause appears in the Spec of $\&$, a coordinating head, and the second one in the complement position of \&.18) At this point, I temporarily assume that the coordination does not require any preferred order (see also (14) from English), which I return to later. Thus, the relative head alone raises to a higher position in (26) to be separated from RCs. ${ }^{19}$ ) This derivation is roughly illustrated below (the element -ass, regarded as a Past tenseelement in the traditional studies, is treated as an Asp(ectual) element, as argued in Yang 2008).

18) In (26) another category like CP may be posited in place of \&P. This depends on whether the coordinator \& projects its label or not, which I leave open here. Again, the number of multiple RCs may be more than two. As aforementioned, Kim (2016: $189,(27))$ offers a cartographic structure in which each relative clause appears in the Spec of a certain functional head under the head-first structure, which is different from the current one, though.

19) Similarly, Ko (2014) proposed a concatenation-cum-sideward movement analysis under the head-last structure; that is, (i) concatenation: $\mathrm{S} \mathrm{V}^{\wedge}[[\mathrm{RC} \cdots]$ [Det NP]], (ii) sideward movement: $\mathrm{S}[$ Det $\mathrm{NP}] \mathrm{i} \mathrm{V} \wedge[[\mathrm{RC} \cdots]$ ei]. The current analysis captures the essence of it without assuming a process like sideward movement. 
(27) a. [MP -e [AspP -ass [VP [V nathana-]]]]

b. [vP [[ku salam][RC \& RC]] [v nathana-ass-e [VP [V nathana-]]]]

c. [TP [ku salam]-i [vP [ [ku salam][RC \& RC]] [v nathana-ass-e [VP [V nathana-]]]]]

d. [TP [ku salam]-i [T' [T nathana-ass-e] [vP [ [ku salam][RC \& RC]] [v nathana-ass-e[VP [V nathana-]]]]]]

In (27b) the inflected verbal complex is formed via head movement of the verb up to $\mathrm{M}$ (ood) through Asp(ect) from (27a) (see (20)); in (27c) the internal subject raises to Spec TP, thereby stranding the RCs behind. To further derive the RDed RCs in $(7 a, b)$, repeated above, the verbal complex raises to a higher functional head $\mathrm{T}$, as seen in (27d). Thus, relative clauses appear separated from the pronounced subject copy in Spec TP, as seen in $(7 a, b){ }^{20)}$

Other usual hypotheses for restrictive RCs, for example, the standard analysis rephrased under the DP system (cf. Chomsky 1977: [DP [D the [NP [NP man] [CP Op $p_{i}\left[C^{\prime}\right.$ that [I met $t_{i}$ yesterday]]]]]]) and the head-raising hypothesis (cf. Kayne 1994: [DP the [CP [NP man $]_{k}\left[C^{\prime}\right.$ that [I met $t_{\mathrm{k}}$ yesterday]]]]), do not actually allow the separation in question; the and man do not form a constituent. Rebuschi (2005: 446-448) points out the compositionality problem with the above two analyses as well as the adjunction analysis; that is, they do not provide Conjunction in the syntactic input given that the relative head and the relative clause are interpreted in terms of Conj(unction) (e.g., [the boy who is smart] $=x[$ boy $(x) \&$ smart(x)]). Thus, as suggested in (28), following Rebuschi (2005: 449, (8)), a conjunctive category, X, may be posited to take the postnominal $\mathrm{RCs}$ as its complement, and X projects XP that hosts the head NP in itsSpec and in turn complements the determiner head $\mathrm{D}$.

(28) $[\mathrm{DP}[\mathrm{D} \mathrm{ku}[\mathrm{XP}[\mathrm{NP}$ salam] [X' $[\mathrm{X}[\& \mathrm{P}[\mathrm{RC} \& \mathrm{RC}]]]]]] \quad(\mathrm{X}=\mathrm{Conj})$

Although this structure may avoid the compositionality problem, the sepa-

20) Chung (2016) provided a generalization that a right-dislocated RC can be associated with a displaced focused relative head (otherwise, with a proximate one), with no exact syntactic structure assumed. The current analysis can directly capture the gist of it - the relative head raises via focus movement stranding the RC behind. 
ration fact, manifested in (7), still remains to be captured.21) On the other hand, the current adjunction analysis in (26), despite the compositionality problem, is factually motivated and allows the separation at issue.

Next, I return to the free ordering in the RDCs in $(7 a, b)$. In what follows, I show that Larson and Takahashi (2007) fail to account for this order. They discussed free ordering in English, as seen in (14), repeated below.

(14) a. the person [who smokes][who I met] (individual $>$ stage) b. the person [who I met][who smokes] (stage $>$ individual)

According to Larson and Takahashi (2007: (49)), the relative head nominal, composed of NP and DP, consists of two prenominal modification domains in languages such as Chinese, Japanese, and Korean--the inner modifier reflects NP-modification and the outer modifier DP-modification. ${ }^{22}$ )

(29) $[\mathrm{DP} \beta[\mathrm{NP} \Gamma[\mathrm{a} N]]] \quad(a=\mathrm{NP}$-modifier, $\beta=$ DP-modifier $)$

They proposed that NP always contains a generic quantifier $\Gamma$ whose scope is limited to NP. Thus, amodifiers inside NP will have potential generic/i-level interpretations, while Bmodifiers outside the scope of the generic quantifier will not have such readings. Thus, it follows that the S-level RC precedes the I-level RC in prenominal RCs in languages such as Chinese, Japanese, and Korean, as seen in the Korean examples, repeated from (17).

21) But this structurewill be needed for multiple prenominal RCs in section 3.2.2.

22) Their original structure contains another $\beta$ in the right periphery of DP, as seen in (i).

(i) $[\mathrm{DP} \beta[\mathrm{NP} \Gamma[\mathrm{a} \mathrm{N}]] \beta]$

( $\alpha=$ NP-modifier, $\beta=$ DP-modifier $)$

But since a DP-modifier cannot actually appear in this position, as seen in (25b), I eliminated it in (29). As aforementioned, an I-level NP-modifier can also precede the determiner. What matters is the ordering: an S-level RC precedes an I-level RC.Thus, a more elaborated structure consistent with the facts observed so far would be like the following.
a. [DP $\beta$ D $\beta[N P \Gamma[a \mathrm{~N}]]]$
( $\alpha=$ NP-modifier, $\beta=$ DP-modifier)
b. [DP $\beta$ a D [NP $\Gamma[$ a $\mathrm{N}]]]$
$(\alpha=$ NP-modifier, $\beta=$ DP-modifier $)$ 
(17) a. [Swunhi-ka ecey mannan][khi(-ka) khun] salam-i nathana-ass-e.

S-Nom yesterday met height-Nom tall man-Nom appear-Past-Dec

'The person who is tall who Swunhi met yesterday appeared.'

b. ?*[Khi(-ka) khun][Swunhi-ka ecey mannan] salam-i nathana-ass-e. height-Nom tall S-Nom yesterday met man-Nom appear-Past-Dec

'Lit. Person who is tall who Swunhi met yesterday appeared.'

They also proposed that postnominal I-level RCs in English have their own source of generic quantification independent of NP, as represented in $(30 a, b)$, and thus, they need not be proximate to the head.

(30) a. the [NP $\Gamma$ person] [ $\Gamma$ who smokes][who I met] b. the [NP $\Gamma$ person] [who I met] [ $\Gamma$ who smokes]

If we followed Larson and Takahashi, I-level RCs in Korean would also include their own generic quantifier in $(7 \mathrm{a}, \mathrm{b})$, represented as $(31 \mathrm{a}, \mathrm{b})$ below.

(31) a. ku [ $\Gamma$ salam]-i nathana-ass-e [ $\Gamma$ khi(-ka) khun][Swunhi-ka ecey mannan]

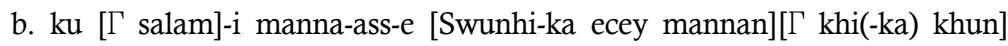

Apparently, this would yield the free ordering effects in question.

However, problems with this analysis are revealed. Reconsider (16) and (17), repeated as (32) and (33), respectively, with the generic quantifier specified.

(32) a. [Swunhi-ka ecey mannan] $[\Gamma$ salam]-i nathana-ass-e $[\Gamma$ khi(-ka) khun] b. ?*[Гkhi(-ka) khun][Г salam]-i nathana-ass-e [Swunhi-ka ecey mannan]

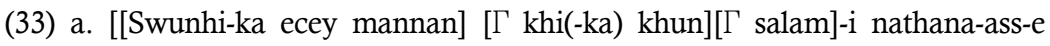
b. ?*[Tkhi(-ka) khun] [Swunhi-ka ecey mannan] [Г salam]-i nathana-ass-e

Now that the I-level RC has its own generic quantifier, it can be separated from the relative head noun, as instantiated in (32a). In (33b), however, this is not possible. In (33a), the I-RC is allowedin proximity to the relative head noun. In (32b), however, this is not possible. Recall that the surface linear order condition in (3), forcing the S-level > I-level order only, should not rule out $(32 b, 33 b)$ since the same I-level $>$ S-level order is allowed in (31a). 
As for the ordering restriction in the prenominal position in examples like (17a,b) (see (33a,b)), Larson and Takahashi (2007: fn. 14) claim that the prenominal I-level RC in Japanese (and thus Korean by implication) isdefective in that it has the reduced/participial status, and that it does not form a larger enough domain to include a generic quantifier.23) So the defective I-level RC cannot be separated from the head and must be proximate to it, as shown in the contrast in $(34 a, b)$ (from $(33 a, b)$ ), with the generic quantifier in the I-level RC eliminated.

(34) a. [Swunhi-ka ecey mannan][khi(-ka) khun] [ $\Gamma$ salam]-i nathana-ass-e b. ?*[khi(ka) khun] [Swunhi-ka ecey mannan] [Гsalam]-i nathana-ass-e

Unfortunately, this is not of any help for $(16 a, b)$, repeated as $(35 a, b)$ from $(32 a, b)$, with the generic quantifier in the I-level RC eliminated. Although the right-dislocated I-level RC is separated from the relative head noun in (35a), the result is good. Although the prenominal I-level $\mathrm{RC}$ is proximate to the relative head noun in (35b), the result is bad.

(35) a. [Swunhi-ka ecey mannan][Г salam]-i nathana-ass-e [khi(-ka) khun] b. ?*[khi(-ka) khun][Г salam]-i nathana-ass-e[Swunhi-ka ecey mannan]

This problem will be resolved in section 3.3.

\subsubsection{Prenominal Orders}

In this section, I offer derivation of the prenominal orders in (17), repeated below (see also (33), (34))

(17) a. [[Swunhi-ka ecey mannan] [khi(-ka) khun][salam]-i nathana-ass-e b. ?*[khi(-ka) khun] [Swunhi-ka ecey mannan] [salam]-i nathana-ass-e

The above orders of multiple prenominal RCs are obtained by raising the multiple postnominal RCs to the prenominal position under the current

23) Unlike in Larson and Takahashi (2007), however, this does not necessarily mean that relative clauses in Korean have a reduced status, for the relative operator is involved, as seen in (26) under the current approach. 
head-first approach. But it needs to be elaborated how the postnominal $\mathrm{RCs}$ raise to become prenominal. Recall also that the surface linear order condition in (3) irrelevant here.

I suggested before that RCs are adjoined to the DP, as represented in (26). However, this adjunction structure is not likely to derive the prenominal RCs in that the adjunct RCs cannot left-adjoin to [DP ku salam] or move into this DP by its lowering. This points to a return to Rebuschi's (2005) structure explored in (28), repeated as (36), with X now specified as Conj(unction).

(36) [DP [D ku [ConjNP [NP salam] [Conj' [Conj [\&P [RC \& RC]]]]]]

Then multiple prenominal RCs are derived by the movement of the postnominal RCs, as shown below, yielding the relevant part in the example in $(17 \mathrm{a}){ }^{24)}$

(37) a. [DP [D [ConjNP [NP [N salam]] [Conj' [Conj [\&P [Swunhi-ka ecey mannan] \& [khi(-ka) khun]]]]]]]

b. [DP [[Swunhi-ka ecey mannan]\&[khi(-ka) khun]] [D [ConjNP [NP [N salam]] [Conj' [Conj [\&P [Swunhi-ka eeey mannan] \& [khi(-ka) khun]]]]]]] (=> (17a))

The example (17b) is derived in the same manner as in (37a,b), as illustrated below.

(38) a. [DP [D [ConjNP [NP [N salam]] [Conj' [Conj [\&P [khi(-ka) khun] \& [Swunhi-ka ecey mannan]]]]]]]

24) When \&P moves over the NP in Spec, ConjNP, a minimality problem may arise. For this, technically, the head Conj may be assumed to raise to the higher head D, so that this movement is allowed due to equi-distance created by this head movement. I provide a relevant case in point from Chinese.

(i) a. [DP D [ConjNP [NP ren][Conj' [Conj de [CP ni zui xihuan]]]]] man DE you most like b. [DP [CP ni zui xihuan] $]_{i}\left[D d_{j}\right.$ [ConjNP [NP ren][Conj' [Conj $\left.\left.\left.\left.t_{j} t_{i}\right]\right]\right]\right]$ c. [ni zui xihuan] de ren 'the man you like the most'

In (ib), de, regarded as a Conj element (cf. Rebuschi 2005), raises to D to derive the surface order in (ic), with the RC moved to Spec, ConjNP. 
b. [DP [[khi(-ka) khun] \& [Swunhi-ka ecey mannan]] [D [ConjNP [NP [N salam]] [Conj' [Conj [\&P [khi(-ka) khun] \& [Swunhi-ka ecey mannan]]]]]] (=> (17b))

Under the current approach, the I-level RC $>$ S-level order in (38a) is allowed, and thus, its prenominal order in (38b) is also expected to be allowed. However, the surface of (38b) is bad, as seen in (17b), repeated above. Compared to (17b), the same order is possible, as attested in (4b), repeated below, if the determiner $k u$ 'the' precedes the I-level RC.25)

(4) b. [Ku [khi(-ka) khun][Swunhi-ka ecey mannan] salam]-i the height-Nom tall S-Nom yesterday met person-Nom nathana-ass-e. appear-Past-Dec

'The person who is tall who Swunhi met yesterday appeared.'

In passing, the surface of $(4 \mathrm{~b})$ can be derived by raising the determiner $k u$ in the $\mathrm{D}$ position from (38b) to a higher functional head of another same type, say, $d$, as seen below.

(39) $\left[\mathrm{d} \mathrm{ku} \mathrm{j}_{\mathrm{j}}\right.$ [DP [[khi(-ka) khun] \& [Swunhi-ka ecey mannan]] [D t $\mathrm{t}_{\mathrm{j}}$ [ConjNP [NP

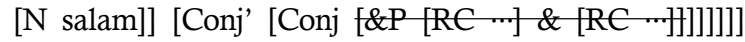

I will account for thecurious contrast between (4b) and (17b) in section 3.3.

Recall that the determiner $k u$ 'the' must appear on the relative head nominal in the right-dislocated RCs (see (7) vs. (8)) and in the prenominal RCs (see (2) vs. (4), (5b) vs. (6)); it may not appear in the prenominal $\mathrm{RCs}$ in (16a, 17a); it can appear after the prenominal $\mathrm{RC}(\mathrm{s})$, as illustrated in $(40,41)$ below.

(40) a. [Swunhi-ka ecey mannan] ku salam-i nathana-ass-e [khi(-ka) khun]. S-Nom yesterday met the person-Nom appeared height-Nom tall 'The person who Swunhi met yesterday appeared who is tall.'

25) The RC after a determiner element is often called a 'descriptive' RC in the Chinese literature, and it is often thought to carry a generic/i-level interpretation in the sense of Larson and Takahashi (2007) (see Del Gobbo 2005: 293 for relevant discussion). But the second RC in (4b) has only stage-level/episodic reading (see also Lin 2008, (15a) for the same fact from Chinese). 
b. [Khi(-ka) khun] ku salam-i nathana-ass-e [Swunhi-ka ecey mannan]. height-Nom tall the person-Nom appeared S-Nom yesterday met 'The person who is tall appeared who Swunhi met yesterday.'

(41) a. [Swunhi-ka ecey mannan][khi(-ka) khun] ku salam-i nathana-ass-e. S-Nom yesterday met height-Nom tall the person-Nom appear-Past-Dec 'The person who is tall who Swunhi met yesterday appeared.'

b. [Khi(-ka) khun][Swunhi-ka ecey mannan] ku salam-i nathana-ass-e. height-Nom tall S-Nom yesterday met the person-Nom appear-Past-Dec 'The person who is tall who Swunhi met yesterday appeared.'

In particular, the ill-formed examples in $(16 \mathrm{~b}, 17 \mathrm{~b})$ are saved by the presence of the determiner $k u$ on the relative head, as shown in (40b, 41b). It appears that the saving effects can be attributed to the fact that this determiner can have a deictic value, or that it can help determine the reference of the relative head noun. ${ }^{26)}$ This will be more elaborated in section 3.3 .

\subsubsection{Split RCs}

I finally turn to derivation of the split RCs, as shown in $(16 a, b)$, repeated below.

(16) a. [Swunhi-ka ecey mannan][salam]-i nathana-ass-e [khi(-ka) khun]

b. ?*[khi(-ka) khun][salam]-i nathana-ass-e [Swunhi-ka ecey mannan]

Under the current approach, the split RC orders in $(16 a, b)$ can beobtained by raising one of the postnominal RCs to the prenominal position and by stranding the other RC behind, as roughly shown in $(42 a, b)$. Thus, the former raising requires the postnominal head structure in (36) and the latter stranding requires the adjunction structure in (26). As illustrated in $(42 a, b)$, the combination of these two structures eventually yields split orders in $(16 a, b) .27)$

26) The determiner following the $\mathrm{RC}$ is often treated as an anaphoric element onto the preceding RC (Del Gobbo 2005: 297 in Chinese). It is, however, seen that it can also be deictic in examples like $(40 \mathrm{~b}, 41 \mathrm{~b})$.

27) Here, the movement of an $\mathrm{RC}$ out of the coordinated structure, [\&P RC1 [\&' [\& $\mathrm{RC} 2]]$, is not considered since it leads to a violation of the CSC. If the structure is cartographic, there arises a violation of the Adjunct Island condition. 
(42) a. [TP [[Swunhi-ka ecey mannan] salam]-i [T' [T nathana-ass-e] [vP [[Swunhi-ka ecey mannan $]_{\mathrm{i}}$-salam] $\mathrm{t}_{\mathrm{i}}$ [ [khi(-ka) khun]] [v nathana-ass-e [VP [V nathana-]]]]]] (=> (16a))

b. [TP [[khi(-ka) khun] salam]-i [T' [T nathana-ass-e] [vP [fkhi(-ka) khun] $]_{i}$ salam] $t_{i}$ [Swunhi-ka ecey mannan]] [v nathana-ass-e [VP [V nathana-]]]]] (=> (16b))

The result, however, diverges. While (42a) produces a good result, (42b) does a bad result. Thus, another curious contrast arises. In addition, next discussion is devoted to accounting for the contrast between (7a) and $(16 b, 17 b) .(7 a)$ is repeated below for the ease of reference.

(7) a. Ku salam-i nathana-ass-e [khi(-ka) khun] [Swunhi-ka ecey mannan]. the person-Nom appeared height-Nom tall S-Nom yesterday met 'The person appeared who is tall who Swunhi met yesterday.'

\subsection{Restricting Relative Clauses}

Having seen that Larson and Takahashi's (2007) basic idea of the generic quantifier $\Gamma$ fails, I newly propose that DP contains a deictic quantifier $\Psi$ whose scope ranges over the whole DP (see Larson and Takahashi 2007: 3.2. for notions deictic vs. generic).

\section{(43) $[\mathrm{DP} \Psi[\mathrm{NP} \Gamma[\mathrm{N}]]]$}

Under the current approach, the adjunction structure in (26) can be re-represented as in (44a), and the absence of the determiner $k u$ in (44b) yields badness. Raising the head nominal results inthe right-dislocated RCs, as represented in $(45 a, b)$ (see also $(27))$.

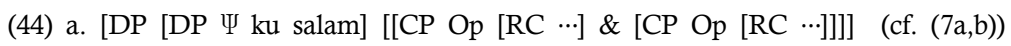

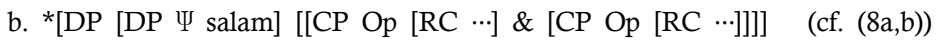

(45) a. [TP [ku salam]-i [T' [Tnathana-ass-e] [vP fku salam]+[RC \& RC] $[\mathrm{v} \cdots[\mathrm{VP}[\mathrm{V} \cdot \cdot]]]]]] \quad($ see $(7 \mathrm{a}, \mathrm{b}))$

b. *[TP [salam]-i [T' [T nathana-ass-e] [vP [ku salam]+[RC \& RC] $[\mathrm{V} \cdots[\mathrm{VP}[\mathrm{V} \cdot \mathrm{*}]]]]]] \quad($ see $(8 \mathrm{a}, \mathrm{b}))$ 
The contrast in (45) indicates that the determiner $k u$ 'the' is crucially involved in (45). This in turn implies that in order for the relative head noun to be restricted by the right-dislocated adjunct $\mathrm{RC}(\mathrm{s})$, the deictic quantifier $\Psi$ requires the presence of a determiner element, as represented in (44) and as manifested in (7a,b). I suggestthat the deictic quantifier $\Psi$ beactivated by a determiner element. In (44a) this deictic quantifier is activated by the determiner $k u$, so that the relative head $k u$ salam 'the person' can be sufficiently deictic or referential for it to be restricted by the right-dislocated RCs, as seen in (45a). In (44b), this quantifier lacking the deictic value is not activated, and thus, the relative head is not properly restricted by the right-dislocated RCs, as seen in (45b). The contrast between (7) and (8) then follows. It is also seen that the activated deictic quantifier, once it thereby gets the deictic value, is not concerned with fixing the particular order between the right dislocated RCs, as seen in $(7 a, b)$.

Next, the prenominal RCs in $(17 a, b)$ can be derived from the base postnominal RCs by raising the whole postnominal \&P to Spec, DP, as shown in $(37,38)$, with a contrasting result, as simply represented in $(46 a, b)$.

(46) a. [DP [\&P [S-RC \& I-RC]] $\Psi$ [D [XP [NP salam] [X' [X ti t]]]] b. ?*[DP [\&P [I-RC \& S-RC]] $\Psi$ [D [XP [NP salam] [X' [X tij]]]]

Under the current approach, the above contrast indicates that while the raised [\&P [S-RC \& I-RC] in (46a) can activate the deictic quantifier $\Psi$, the raised [\&P [I-RC \& S-RC]] in (46b) does not. This will follow if the former $\& \mathrm{P}$ is regarded as stage level as the S-RC is in Spec, \&, and if the latter \&P is regarded as individual level as the I-RC is in Spec, $\&$. Given Spec-head agreement, the whole \&P would assume the properties of its Spec. Thus, the deictic quantifier $\Psi$ can be activated by the S-level $\mathrm{RC}$ in (46a), but it cannot by the I-level RC in (46b) in that while the generic quantifier $\Gamma$ is associated with generic/i-level interpretations (Larson and Takahashi 2007), the deictic quantifier $\Psi$ is associated with potential deictic/s-level interpretations. If the determiner $k u$ 'the' is present in (46b), it can help activate the deictic quantifier $\Psi$, as represented in (47) and manifested in (4b). 
(47) $\left[\mathrm{ku}\left[\mathrm{DP}[\& \mathrm{P}[\mathrm{I}-\mathrm{RC} \& \mathrm{~S}-\mathrm{RC}]]_{\mathrm{i}} \Psi\left[\mathrm{D}\left[\mathrm{XP}\left[\mathrm{NP}\right.\right.\right.\right.\right.$ salam] [X' $\left.\left.\left.\left.\left[\mathrm{X} \mathrm{t}_{\mathrm{i}}\right]\right]\right]\right]\right]$

Finally, the contrast in split RCs, as realized in $(5 a, b)$, also follows from the current proposal, as represented below.

(48) a. [TP [[S-RC] $\Psi$ salam]-i [nathana-ass-e [vP [[S-RC $]_{\mathrm{i}} \Psi$ salam] $\mathrm{t}_{\mathrm{i}}$ [I-RC]] $[\mathrm{v} \cdots[\mathrm{VP}[\mathrm{V}-\boldsymbol{- r}]]]]] \quad(=>(5 \mathrm{a}))$

b. ?*[TP [[I-RC] $\Psi$ salam]-i [nathana-ass-e [vP [[I-RC] $]_{*} \Psi$ salam] $\left.\left.\mathrm{t}_{\mathrm{i}}\right][\mathrm{S}-\mathrm{RC}]\right]$ $[\mathrm{v} \cdots[\mathrm{VP}[\mathrm{V}-\cdots]]]]] \quad(=>(5 \mathrm{~b}))$

In (48a), the deictic quantifier $\Psi$ can be activated by the single S-RC, and the right-dislocated I-RC can restrict the relative head noun. In (48b), however, the deictic quantifier $\Psi$ cannot be activated by the I-RC, and the right-dislocated S-RC cannot restrict the relative head noun, either. Again, the addition of the determiner $k u$ 'the', rendering the deictic quantifier $\Psi$ activated, saves (48b), as shown below and manifested in (6).

(49) $\left[\mathrm{ku}[\mathrm{I}-\mathrm{RC}] \Psi\right.$ salam]-i [nathana-ass-e $\left[\mathrm{vP}\left[[\mathrm{I}-\mathrm{RC}]_{\mathrm{i}}-\Psi\right.\right.$ salam $\left.\left.] \mathrm{t}_{\mathrm{i}}\right\}+[\mathrm{S}-\mathrm{RC}]\right]$ $[\mathrm{v} \cdots[\mathrm{VP}[\mathrm{V}-\cdots]]]]]$

Under the current approach, the following example containing a single I-level RC also needs to be discussed.

(50) [Khi(-ka) khun] salam-i nathana-ass-e. height-Nom tall person-Nom appeared 'Lit. Person who is tall appeared.'

It seems that the subject with an I-level RC in (50) is rather insufficient in referring to a specific/non-generic person in the discourse. If a determiner like $k u$ 'the' or han 'a/one' is added, as seen in (51), the activation of the deictic quantifier $\Psi$ can be clearly made.28)29)

28) In $(51 \mathrm{~b}, \mathrm{~d})$, the I-level RC appears in the domain of the deictic quantifier, which is not captured in Larson and Takahashi (2007).

29) The same holds of the example in (5b) as well. This example is bad, and it becomes good if the determiner ku 'the' precedes the RCs, as shown in (6). 
(51) a. [ Ku [khi(-ka) khun] salam]-i nathana-ass-e. the height-Nom tall man-Nom appeared 'The person who is tall appeared.'

b. [[Khi(-ka) khun] ku salam]-i nathana-ass-e. height-Nom tall the man-Nom appeared

'The person who is tall appeared.'

c. ?[Han [khi(-ka) khun] salam]-i nathana-ass-e. one height-Nom tall man-Nom appeared

'A person who is tall appeared.'

d. [[Khi(-ka) khun] han salam]-i nathana-ass-e. height-Nom tall one man-Nom appeared

'A person who is tall appeared.'

In fact, the discussion has been made with the stage level predicate nathana'appear' in the examples considered so far. In this light, if the subject with an I-level RC in (50) lacks the deictic value, it may be said that this generic/i-level subject is not concomitant with the stage level predicate in the sentence. On the other hand, in (51) the subject now has the deictic quantifier due to the presence of a determiner, and thus, it can be concomitant with the stage level predicate in the sentence.

On the other hand, if the predicate is not stage level but individual level, for example, singkep- 'insipid, uninteresting', as seen in (52), it is expected that this deictic quantifier is not required.

(52) a. [Khi(-ka) khun] salam-i singkep-e. height-Nom tall man-Top uninteresting-Dec 'Lit. Person who is tall is uninteresting.'

b. [Khi(-ka) khun] salam-un singkep-e. height-Nom tall man-Top uninteresting-Dec 'Lit. Person who is tall is uninteresting.'

In (52a), the subject, [[khi(-ka) khun] salam]-i, has a generic/exhaustive reading; in (52b) it has a generic reading. In both cases, indeed, deictic reading is absent. As Larson and Takahashi (2007: (49)) propose, if NP 
always contains a generic quantifier $\Gamma$, the I-level RC in (52) may be said to activate the generic quantifierunder the current approach.On the other hand, an S-level RC can appear with the individual level predicate, as seen below.

(53) [Swunhi-ka ecey mannan] salam-i/un singkep-e.

S-Nom yesterday met man-Nom/Top uninteresting-Dec

'The person who Swunhi met yesterday is uninteresting.'

The subject has a deictic reading. Since the deictic quantifier ranges over the whole DP involving the generic quantifier inside, it may not be inconsistent with the individual level predicate.

\section{Summary and Conclusion}

In this paper, I discussed three types of multiple relative clauses available in Korean, that is, prenominal, right-dislocated, and split relative clauses. In split relative clauses, one relative clause (RC) is prenominal and the other is right dislocated. I attempted to account for ordering restrictions among those RCs and offer plausible derivations of them. In the prenominal and split RCs, a stage level RC precedes an individual level RC in Larson and Takahashi's (2007) term; in the right-dislocated RCs, free order obtains. Interestingly, an individual level RC may precede a stage level RC in the prenominal position if a determiner like $k u$ 'the' precedes the initial I-level RC. As this part of the facts is particularly different from that in Chinese, as reported in Del Gobbo (2005), Lin (2008), among others, it requires a different analysis. Departing from Larson and Takahashi (2007) and Del Gobbo (2005), I newly proposed that a deictic quantifier be activated, which is assumed to be required to determine the reference of the relative head noun. This activation can be done by a determiner element or a stage level RC in the prenominal position, but not by an individual level RCon the ground that while a stage-level RC is associated with deictic interpretation, an individual-level $\mathrm{RC}$ is associated with only generic interpretation. I also showed that differ- 
ent orders of RCs in the three types of multiple RCs follow from the present proposal. In deriving the three types of multiple RCs, I proposed three distinct ways of forming RCs in Korean: (i) restrictive RCs (RRCs) are adjoined to DP as CPs for right-dislocated RCs, (ii) the head NP is base-generated in Spec, XP and RRCs are complement of X for prenominal RCs (X can be Conjunction in the sense of Rebuschi 2005), (iii) combination of (i) and (ii) for split RCs. There are a few important conclusions drawn from the discussions in the paper. The explanation of the distribution of multiple RCs in Korean can be plausibly offered under the single clause approach. Further, it requires the head-first structure for Korean. The traditional head-final hypothesis, coupled with either the single clause approach with rightward/leftward movement or the double clause approach with leftward movement and deletion, does not properly derive the multiple relative constructions considered here.

\section{References}

Aoun, Joseph and Y.-H. Audry Li. (2003). Essays on the representational and derivational nature of grammar: the diversity of wh-constructions. Cambridge, MA: MIT Press.

Bach, Emmon and Robin Cooper. (1978). The NP-S analysis of relative clauses and compositional semantics. Linguistics and Philosophy 2, 145-150.

Chomsky, Noam. (1977). On wh-movement. In P. W. Culicover, T. Wasow and A. Akmajian (eds.). Formal syntax, 71-132. New York, Academic Press. (2008). On phases. In Foundational issues in linguistic theory: Essays in honor of Jean Roger Vergnaud, ed., by Robert Freidin, Carlos P. Otero \& Maria Lisa Zubizarreta, pp. 133-166. Cambridge, MA: MT Press.

Chung, Daeho. (2009). An elliptical coordination analysis of the right dislocated construction in Korean. The Linguistic Association of Korea Journal 17.4, 1-23.

(2016). On what determines the modification relation in Korean adnominal adjunct RDCs. Language Research 33.3, 351-370.

Del Gobbo, Francesca. (2005). Chinese relative clauses: restrictive, descriptive or appositive? In Proceedings for The XXX Incongtro di Grammatica Generativa, eds. L. Bruge, G. Giusti, N. Munaro, W. Schweikert, and G. Turano, 287-305. Venezia: Cafoscarina. 
Kayne, Richard. (1994). The antisymmetry of syntax. Cambridge, MA: MIT Press. Kim, Minjoo. (2014). Adjective ordering: A view from Korean. Proceedings of the 31th West Coast Conference on Formal Linguistics, 285-294.

(2016). Cognitive indexical usage of demonstrative $k u$ in Korean and a split DP analysis. Proceedings of the 18thSeoul International Conference on Generative Grammar: Beyond core syntax: A minimalist approach, eds. By Tae Sik Kim and Seungwan Ha, 175-194.

Kim, Sun-Woong and Myung-Kwan Park. (2010). Parallel linearization: A thought on afterthought. The Journal of Studies in Linguistics 26.3, 459-480.

Kim, Young-Kook. (1996). On the structure of relative clauses. In Proceedings of the 1996 Seoul International Conference on Generative Grammar: Morphosyntax in generative grammar, eds. By H-D Ahn, Y-S Kim, and S Lee, 251-277.

Ko, Heejeong. (2014). Remarks on right dislocation construction in Korean: Challenges to bi-clausal analyses. Language Research 50.2, 275-310.

Larson, Richard K. and Naoko Takahashi. (2007). Order and interpretation in prenominal relative clauses. Proceedings of the Workshop on Altaic Formal Linguistics II. MIT Working Papers in Linguistics Vol. 54. eds. By M. Kelepir and B. Ozturk, 101-120. Cambridge, MA: MITWPL.

Lee, Jeong-Shik. (1997). Antecedent-contained deletion constructions in Korean. Harvard Studies in Korean Linguistics 7, 399-413.

(2009). A verb-initial single clause analysis for right-dislocated constructions in Korean. Studies in Modern Grammar 57, 127-157.

(2010). Why only predicate-final in embedded clauses in Korean? Studies in Modern Grammar 61, 99-138.

(2011a). Some loopholes of the double clause approach to the right dislocated construction in Korean. Studies in Modern Grammar 63, 113-146. Grammar 65, 45-72.

(2011b). Layered VP and inner aspect. Studies in Modern

Lin, Jo-wang. (2008). The order of stage-level and individual-level relatives and superiority effects. Language and Linguistics 9.4, 839-864.

Park, Myung-Kwan and Sung-Woong Kim. (2009). The syntax of afterthoughts in Korean: Move and Delete. The Linguistic Association of Korea Journal 17.4, 25-53.

Rebuschi, Georges. (2005). Generalizing the antisymmetric analysis of coordination to nominal modification. Lingua 115, 445-459.

Safir, Ken. (1986). Relative clause in a theory of binding and levels. Linguistic Inquiry 17, 663-689.

Yang, Jeong-Seok. (2008). The morphosyntax of temporal elements in Korean. Korean Journal of Linguistics 33, 693-722. 
Jeong-Shik Lee

Department of English Language and Literature

Wonkwang University

460 Iksandae-ro, Iksan, Jeonbuk, 54538, South Korea

E-mail: jslee@wku.ac.kr

Received: June 29, 2016

Revised version received: October 16, 2017

Accepted: November 20, 2017 
